\title{
Periodic waves in unsaturated porous media with hysteresis *
}

\author{
Bettina Albers $^{\dagger} \quad$ Pavel Krejčí Elisabetta Rocca $^{\ddagger}$
}

September 13, 2018

\begin{abstract}
We consider a PDE system with degenerate hysteresis describing unsaturated flow in $3 \mathrm{D}$ porous media. Assuming that a time periodic forcing is prescribed on the boundary, we prove that a time periodic response exists as long as the amplitude of the forcing terms is small enough to keep the solution within the convexity domain of the hysteresis operator.
\end{abstract}

\section{Introduction}

Periodic waves of a given frequency are often used in non-destructive testing of porous media. In particular, building materials, geomaterials, tissues, nanomaterials etc. are examples of porous materials, in which non-destructive testing methods are of central importance. The reason is not only that the sample does not have to be destroyed or invaded, but another advantage in comparison with conventional methods is that non-destructive testing is mostly cost-saving. For example, this is the case of testing of soils, where procedures exploiting the properties of acoustic waves are cheaper than drilling the boreholes.

There are many different techniques of non-destructive testing: ultrasonic methods, magnetic particle inspection, liquid penetrant inspection, electrical measurements or radiography - for details see, e. g., [24, 25, 11]. For further information on acoustic methods of non-destructive testing see, e. g., [16, 19].

In the geotechnical field, the wave analysis of both body and surface waves ([5]) may lead to the construction of several non-destructive testing methods. Body or bulk waves travel through the

\footnotetext{
*Supported by the GAČR Grant GA15-12227S, RVO: 67985840, FWF P23628-N18, and by the FP7-IDEASERC-StG \#256872 (EntroPhase)

$\dagger$ University of Duisburg-Essen, Faculty of Engineering, Department of Civil Engineering, 45117 Essen , Germany, E-mail bettina.albers@uni-due.de.

${ }_{\ddagger}^{\ddagger}$ Institute of Mathematics, Czech Academy of Sciences, Žitná 25, CZ-11567 Praha 1, Czech Republic, E-mail krejci@math.cas.cz.

$\S$ Dipartimento di Matematica, Università degli Studi di Pavia. Via Ferrata 5, I-27100 Pavia, Italy, E-mail elisabetta.rocca@unipv.it.
} 
interior of a medium, while surface waves propagate along the surface of a body or along the interface of two media. The amplitudes of surface waves decay in the direction perpendicular to the surface so fast that they can be assumed to be zero in the depth of a few wavelengths. The analysis of surface waves in saturated porous media ([1]) may help to develop a method for soil characterization. By use of the SASW-technique (Spectral Analysis of Surface Waves), e. g., [23. 15], conclusions about building grounds can be drawn from the measurement of sound wave speeds. In other words, expensive and invasive acoustic measurements in boreholes or laboratory tests are not necessary to characterize the soil prior to a building project. In SASW tests, two or more receivers are placed on the surface, and a hammer (or a signal with a certain frequency) is used to generate surface waves whose speeds are recorded. Algorithms based on the Fast Fourier Transform applied to the acquired data then produce a stiffness versus depth plot. While in the classical method the wave propagation in single-component media is analyzed, in [1] the considerations are extended to two and three component modeling.

If the pores of a porous medium are filled with two (or more) immiscible fluids as, for example, water and air, then they are called partially saturated. The pore fluids possess different partial pressures, i. e. there exists a discontinuity in the pressure across the interface. This difference is called the capillary pressure. It depends on the geometry of the pore space, on the nature of the solids and on the degree of saturation, i. e. the ratio of the volume occupied by one of the pore fluids over the entire pore volume.

For the description of the propagation of sound waves in partially saturated soils (threecomponent media) a linear macroscopic model is introduced in [5]. However, experimental studies of wetting and dewetting curves in partially saturated porous media exhibit strong capillary hysteresis effects, see [17], which are due to the surface tension on the liquid-gas interface. Flynn et al. [13, 14] suggested to model hysteresis phenomena in porous media by means of the Preisach operator originally designed in [22] for magnetic hysteresis in ferromagnetics. Two models to describe processes in partially saturated media are presented in [7]. The first model does not explicitly contain a hysteresis operator and the effect of hysteresis in the capillary pressure curve is accounted for by investigating the two processes drainage and imbibition separately, cf. also [3, 4. The second model is a thermomechanical model involving the Preisach operator, while plastic hysteresis is described in terms of the Prandtl-Reuss model.

In the present paper, we study the mathematical problem of well posedness of the porous medium model proposed in [6] under periodic mechanical forcing. We assume that a time periodic force is prescribed on the boundary of the domain, and look for time periodic mechanical waves in the system of balance equations. The main difference with respect to [6] consists in the hypothesis that the solid matrix material is elastic within the small deformation hypothesis, so that the momentum balance equation is linear. The only nonlinearity in the problem is thus the degenerate Preisach hysteresis operator in the mass balance equation. On the other hand, since viscosity is missing in the model, we lose the higher order a priori estimates, which were used in [6] to control the degeneracy of the Preisach operator. Instead, we make use of the second order energy inequality related to the convexity of small amplitude hysteresis loops to prove that periodic solutions of the system exist provided the amplitude of the external forcing is sufficiently small. Note that for any nonlinear pressure-saturation relation without hysteresis, such a result would be much more difficult to obtain, since no counterpart of the second order energy inequality is available in this case. 
The structure of the paper is as follows. In Section 1, we present the model situation, and in Section 2 we state Theorem 2.1 which is the main Existence Theorem of the paper. Section 3 is devoted to a survey about Preisach hysteresis, and Section 4 contains the proof of Theorem 2.1.

\section{The model}

The present paper deals with the following model for fluid flow in an unsaturated porous solid

$$
\begin{aligned}
\rho_{S} u_{t t}+c u_{t} & =\operatorname{div} \mathbf{A} \nabla_{s} u+\nabla p+f_{0}, \\
G[p]_{t} & =\operatorname{div} u_{t}+\frac{\mu}{\rho_{L}} \Delta p .
\end{aligned}
$$

in a spatial domain $\Omega$ and time $t \in \mathbb{R}$, for unknown functions $u$ (displacement) and $p$ (capillary pressure), with a operator $G$ characterizing the hysteresis dependence between $p$ and the relative air content $A \in[-1,1], A=G[p]$ as in [6], see Figure 1, and with constant coefficients $\rho_{S}, \rho_{L}$ (mass densities of the solid and liquid, respectively), and $\mu$ (permeability). By $\nabla_{s}$ we denote the symmetric gradient, $\mathbf{A}$ is a constant elasticity matrix, and $f_{0}$ is a given external volume force. Eq. (1.1) is the momentum balance, (1.2) is the liquid mass balance.

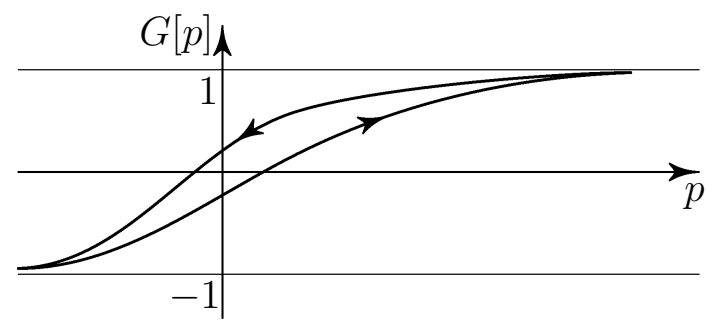

Figure 1: The pressure-saturation hysteresis

On the boundary $\partial \Omega$ we prescribe boundary conditions

$$
\left.\left(u-u^{*}\right)\right|_{\partial \Omega}=0,\left.\quad \nabla p \cdot n\right|_{\partial \Omega}=\gamma(x)\left(p^{*}-p\right),
$$

where $u^{*}$ is a given displacement, $n$ is the unit outward normal vector, $p^{*}=p^{*}(x, t)$ is a given outer pressure, and $\gamma(x) \geq 0$ is a given permeability of the boundary which is positive on a set of positive measure.

A similar system was derived in [6] in the form

$$
\begin{aligned}
& \rho_{S} u_{t t}=\operatorname{div}\left(\mathbf{B} \nabla_{s} u_{t}+P\left[\nabla_{s} u\right]\right)+\nabla p+f_{0}, \\
& G[p]_{t}=\operatorname{div} u_{t}+\frac{1}{\rho_{L}} \operatorname{div}(\mu(p) \nabla p) .
\end{aligned}
$$

as a model for isothermal flow in an unsaturated viscoelastoplastic porous solid, where $P$ is a constitutive operator of elastoplasticity, $\mathbf{B}$ is a constant viscosity matrix, and $\mu(p)$ is a pressure dependent permeability coefficient. In reality, the coefficient $\mu$ should depend on the saturation, that is, on $G[p]$, but the analysis carried out in [8, 9] shows that the presence of the 
hysteresis operator in the coefficient makes the problem difficult, so that it cannot be solved without additional space or time regularization.

The main result of [6] was the proof of existence of a strong solution to the above system with natural boundary conditions and given initial conditions. In particular, it was shown that the solution remains bounded away from the degeneracy of the hysteresis operator $G$.

The system (1.1)-(1.2) differs from (1.4)-(1.5) in several respects. Notice first that the strong viscous dissipative term $\operatorname{div} \mathbf{B} \nabla_{s} u_{t}$ is replaced with a more realistic weaker term $c u_{t}$ corresponding to contact friction on the solid-liquid interface with a constant friction parameter $c>0$. The strong a priori estimates as in [6] resulting from the viscous term are no longer available for (1.1) -(1.2), so that we need additional modeling hypotheses to prove the solvability of the system. More specifically, the solid matrix is assumed elastic, that is, $P\left[\nabla_{s} u\right]=\mathbf{A} \nabla_{s} u$, the permeability coefficient $\mu$ is a positive constant, and we consider time periodic data.

The system is linear in $u$, so that we can replace $u$ by $u-u^{*}$ if $u^{*}$ is sufficiently regular, and reformulate (1.1)-(1.3) as

$$
\begin{array}{r}
\rho_{S} u_{t t}+c u_{t}=\operatorname{div} \mathrm{A} \nabla_{s} u+\nabla p+f, \\
G[p]_{t}=\operatorname{div} u_{t}+\frac{\mu}{\rho_{L}} \Delta p+h, \\
\left.u\right|_{\partial \Omega}=0,\left.\quad \nabla p \cdot n\right|_{\partial \Omega}=\gamma(x)\left(p^{*}-p\right),
\end{array}
$$

with given functions $f$ and $h$.

\section{Statement of the problem}

We assume that all given functions $f, h$, and $p^{*}$ are time periodic with the same period. The values of the physical constants are not relevant for our analysis, so that for simplicity, we consider system (1.6)-(1.8) in the form

$$
\begin{aligned}
u_{t t}+u_{t} & =\operatorname{div} \mathrm{A} \nabla_{s} u+\nabla p+f \\
G[p]_{t} & =\operatorname{div} u_{t}+\Delta p+h \\
\left.u\right|_{\partial \Omega}=0, & \left.\nabla p \cdot n\right|_{\partial \Omega}=\gamma(x)\left(p^{*}-p\right)
\end{aligned}
$$

with $2 \pi$-periodic data, and introduce the notation

$$
L_{2 \pi}^{q}(\Omega)=\left\{y \in L_{l o c}^{q}(\Omega \times \mathbb{R}): y(x, t+2 \pi)=y(x, t) \text { a. e. }\right\},
$$

and similarly for $L_{2 \pi}^{q}(\partial \Omega)$ etc. The norm in $L_{2 \pi}^{q}(\Omega)$ is defined as

$$
\|y\|_{q, \Omega, 2 \pi}=\left(\int_{2 \pi}^{4 \pi} \int_{\Omega}|y|^{q} \mathrm{~d} x \mathrm{~d} t\right)^{1 / q}
$$

and in $L_{2 \pi}^{q}(\partial \Omega)$ we introduce the seminorm

$$
\|y\|_{q, \partial \Omega, 2 \pi, \gamma}=\left(\int_{2 \pi}^{4 \pi} \int_{\partial \Omega} \gamma(x)|y|^{q} \mathrm{~d} s(x) \mathrm{d} t\right)^{1 / q} .
$$


Theorem 2.1. Let $\Omega$ be a bounded domain with $C^{1,1}$ boundary, let $\gamma \in C^{1}(\partial \Omega)$ be a nonnegative function which does not identically vanish, and let the data $f, h, p^{*}$ be such that $f, f_{t}, h, h_{t} \in L_{2 \pi}^{2}(\Omega), p^{*}, p_{t}^{*} \in L_{2 \pi}^{2}(\partial \Omega)$. Set

$$
\delta=\max \left\{\|f\|_{2, \Omega, 2 \pi},\left\|f_{t}\right\|_{2, \Omega, 2 \pi},\|h\|_{2, \Omega, 2 \pi},\left\|h_{t}\right\|_{2, \Omega, 2 \pi},\left\|p^{*}\right\|_{2, \partial \Omega, 2 \pi, \gamma},\left\|p_{t}^{*}\right\|_{2, \partial \Omega, 2 \pi, \gamma}\right\} .
$$

Then there exists $\delta^{*}>0$ such that if $\delta<\delta^{*}$, then system (2.1)-(2.3) has a solution $u, p$ such that $u, u_{t}, u_{t t}, \nabla_{s} u, \nabla_{s} u_{t}$, div $\mathbf{A} \nabla_{s} u, p, p_{t}, \nabla p, \nabla p_{t}, \Delta p \in L_{2 \pi}^{2}(\Omega)$.

The reason why we have to assume that the data are small is related to the fact that higher order a priori estimates, which are not available here due to the absence of the viscosity term $\operatorname{div} \mathbf{B} \nabla_{s} u_{t}$, can only be recovered as long as the input $p$ of the hysteresis operator $G$ stays in the convexity domain of $G$. Details will be given in Section 3 .

\section{Hysteresis operators}

We recall here the basic concepts of the theory of hysteresis operators that are needed in the sequel. The construction of the operator $G$ is based on the variational inequality

$$
\begin{cases}\left|p(t)-\xi_{r}(t)\right| \leq r & \forall t \in[0, T] \\ \left(\xi_{r}(t)\right)_{t}\left(p(t)-\xi_{r}(t)-z\right) \geq 0 & \text { a. e. } \forall z \in[-r, r], \\ p(0)-\xi_{r}(0)=\max \{-r, \min \{p(0), r\}\} . & \end{cases}
$$

It is well known ([18) that for each given input function $p \in W^{1,1}(0, T)$ for some $T>0$ and each parameter $r>0$, there exists a unique solution $\xi_{r} \in W^{1,1}(0, T)$ of the variational inequality (3.1). The mapping $\mathfrak{p}_{r}: W^{1,1}(0, T) \rightarrow W^{1,1}(0, T)$ which with each $p \in W^{1,1}(0, T)$ associates the solution $\xi_{r}=\mathfrak{p}_{r}[p] \in W^{1,1}(0, T)$ of (3.1) is called the play operator, and the parameter $r>0$ can be interpreted as a memory parameter. The proof of the following statements can be found, e. g., in [20, Chapter II].

Proposition 3.1. For each $r>0$, the mapping $\mathfrak{p}_{r}: W^{1,1}(0, T) \rightarrow W^{1,1}(0, T)$ is Lipschitz continuous and admits a Lipschitz continuous extension to $\mathfrak{p}_{r}: C[0, T] \rightarrow C[0, T]$ in the sense that for every $p_{1}, p_{2} \in C[0, T]$ and every $t \in[0, T]$ we have

$$
\left|\mathfrak{p}_{r}\left[p_{1}\right](t)-\mathfrak{p}_{r}\left[p_{2}\right](t)\right| \leq\left\|p_{1}-p_{2}\right\|_{[0, t]}:=\max _{\tau \in[0, t]}\left|p_{1}(\tau)-p_{2}(\tau)\right|
$$

Moreover, for each $p \in W^{1,1}(0, T)$, the energy balance equation

$$
\mathfrak{p}_{r}[p]_{t} p-\frac{1}{2}\left(\mathfrak{p}_{r}^{2}[p]\right)_{t}=\left|r \mathfrak{p}_{r}[p]_{t}\right|
$$

and the identity

$$
\mathfrak{p}_{r}[p]_{t} p_{t}=\left(\mathfrak{p}_{r}[p]_{t}\right)^{2}
$$

hold almost everywhere in $(0, T)$. 
Similarly as above, we define the spaces of $2 \pi$-periodic functions of time

$$
L_{2 \pi}^{q}=\left\{z \in L_{l o c}^{q}(\mathbb{R}): z(t+2 \pi)=z(t) \text { a.e. }\right\}, C_{2 \pi}=\{z \in C(\mathbb{R}): z(t+2 \pi)=z(t) \forall t \in \mathbb{R}\},
$$

endowed with the natural norms

$$
|z|_{q, 2 \pi}=\left(\int_{2 \pi}^{4 \pi}|z(t)|^{q} \mathrm{~d} t\right)^{1 / q}
$$

and similarly for $C_{2 \pi}, W_{2 \pi}^{k, q}$ for $k \in \mathbb{N}$ and $q \geq 1$, etc.

Proposition 3.2. For every $p \in C_{2 \pi}$ and every $r>0$ we have $\mathfrak{p}_{r}[p](t+2 \pi)=\mathfrak{p}_{r}[p](t)$ for all $t \geq 2 \pi$. In particular, by extending $\mathfrak{p}_{r}[p]$ backward from the interval $[2 \pi, \infty)$ periodically to $\mathbb{R}$, we can assume that $\mathfrak{p}_{r}$ maps $C_{2 \pi}$ into $C_{2 \pi}$.

Given a nonnegative function $\rho \in L^{1}((0, \infty) \times \mathbb{R})$, we define the operator $G$ as a mapping that with each $p \in C_{2 \pi}$ associates the integral

$$
G[p](t)=\int_{0}^{\infty} \int_{0}^{\mathfrak{p}_{r}[p](t)} \rho(r, v) \mathrm{d} v \mathrm{~d} r .
$$

Directly from the definition (3.1) of the play, we see with the notation of (3.2) that the implication

$$
r \geq\|p\|_{[0, t]} \Longrightarrow \mathfrak{p}_{r}[p](t)=0
$$

holds for every $p \in W^{1,1}(0, T)$ and every $T>0$ (hence, for every $p \in C_{2 \pi}$ ), so that the integration domain in (3.5) is always bounded.

Definition (3.5) is equivalent to the Preisach model proposed in [22], see [21]. For our purposes, we prescribe the following hypotheses on $\rho$.

Hypothesis 3.3. The function $\rho \in W^{1, \infty}((0, \infty) \times \mathbb{R})$ is such that there exists a function $\rho^{*} \in L^{1}(0, \infty)$ such that for a. e. $(r, v) \in(0, \infty) \times \mathbb{R}$ we have $0 \leq \rho(r, v) \leq \rho^{*}(r)$, and we put

$$
C_{\rho}=\int_{0}^{\infty} \int_{-\infty}^{\infty} \rho(r, v) \mathrm{d} v \mathrm{~d} r, \quad C_{\rho}^{*}=\int_{0}^{\infty} \rho^{*}(r) \mathrm{d} r .
$$

Furthermore, there exists $R>0$ for which the following condition holds:

$$
A_{R}:=\inf \{\rho(r, v): r+|v| \leq R\}>0 .
$$

Put $C_{R}:=\sup \left\{\left|\frac{\partial}{\partial v} \rho(r, v)\right|: r+|v| \leq R\right\}$. Taking $R>0$ smaller, if necessary, we can assume that there exists $K_{R}>0$ such that

$$
\frac{1}{2} A_{R}-R C_{R} \geq K_{R}
$$

From (3.3), (3.4), and (3.5) we immediately deduce the Preisach energy identity

$$
G[p]_{t} p-V[p]_{t}=\left|D[p]_{t}\right| \text { a. e. }
$$


with a Preisach potential $V$ and dissipation operator $D$ defined as

$$
V[p](t)=\int_{0}^{\infty} \int_{0}^{\mathfrak{p}_{r}[p](t)} v \rho(r, v) \mathrm{d} v \mathrm{~d} r, \quad D[p](t)=\int_{0}^{\infty} \int_{0}^{\mathfrak{p}_{r}[p](t)} r \rho(r, v) \mathrm{d} v \mathrm{~d} r .
$$

A straightforward computation shows that $G$ is Lipschitz continuous in $C[0, T]$. Indeed, using (3.2) and Hypothesis [3.3, we obtain for $p_{1}, p_{2} \in C[0, T]$ and $t \in[0, T]$ that

$$
\left|G\left[p_{1}\right](t)-G\left[p_{1}\right](t)\right|=\left|\int_{0}^{\infty} \int_{\mathfrak{p}_{r}\left[p_{1}\right](t)}^{\mathfrak{p}_{r}\left[p_{2}\right](t)} \rho(v, r) \mathrm{d} v \mathrm{~d} r\right| \leq C_{\rho}^{*} \max _{\tau \in[0, t]}\left|p_{1}(\tau)-p_{2}(\tau)\right| .
$$

Following [12], we define the convexified operator $G_{R}$ (see Figure 2) by a formula similar to (3.5)

$$
G_{R}[p](t)=\int_{0}^{\infty} \int_{0}^{\mathfrak{p}_{r}[p](t)} \rho_{R}(r, v) \mathrm{d} v \mathrm{~d} r
$$

where

$$
\rho_{R}(r, v)= \begin{cases}\rho(r, v) & \text { if } r+|v| \leq R, \\ \rho(r,-R+r) & \text { if } v<-R+r, r \leq R, \\ \rho(r, R-r) & \text { if } v>R-r, r \leq R, \\ \rho(R, 0), & \text { if } r>R .\end{cases}
$$

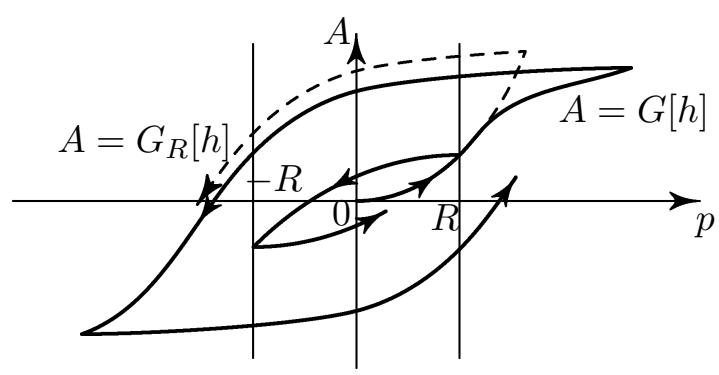

Figure 2: Local behavior of the operator $G$ and its convexification $G_{R}$.

It is shown in [12] that the operator $G_{R}$ satisfies globally the hypotheses of [20, Theorem II.4.19], that is, the ascending hysteresis branches are uniformly convex and the descending branches are uniformly concave, so that for each input $p \in W_{2 \pi}^{2,1}$, the second order energy inequality holds in the form

$$
-\int_{2 \pi}^{4 \pi} G_{R}[p]_{t} p_{t t} \mathrm{~d} t \geq \frac{K_{R}}{2} \int_{2 \pi}^{4 \pi}\left|p_{t}\right|^{3} \mathrm{~d} t .
$$

As a consequence of (3.10) we also have

$$
\int_{2 \pi}^{4 \pi} G_{R}[p]_{t} p \mathrm{~d} t \geq 0
$$


for every $p \in W_{2 \pi}^{1,1}$. Since the density $\rho$ is globally bounded above by a constant $H_{\rho}$ and the implication (3.6) holds, the operator $G_{R}$ has quadratic growth in the sense

$$
\left|G_{R}[p](t)\right| \leq H_{\rho}\|p\|_{[0, t]}^{2}, \quad\left|G_{R}[p]_{t}(t)\right| \leq H_{\rho}\|p\|_{[0, t]}\left|p_{t}(t)\right| \text { a. e. }
$$

for all functions $p \in W^{1,1}(0, T)$ and all $T>0$. On the other hand, we have the implication

$$
|p(t)| \leq R \forall t \geq 0 \Longrightarrow G_{R}[p](t)=G[p](t) \forall t \geq 0 \text {. }
$$

\section{Proof of Theorem 2.1}

We replace the operator $G$ by $G_{R}$ and consider the variational formulation of the convexified version of Problem (2.1)-(2.3)

$$
\begin{aligned}
\int_{\Omega}\left(\left(u_{t t}+u_{t}\right) \phi+\mathbf{A} \nabla_{s} u: \nabla_{s} \phi+p \operatorname{div} \phi\right) \mathrm{d} x & =\int_{\Omega} f \phi \mathrm{d} x \\
\int_{\Omega}\left(\left(G_{R}[p]_{t}-\operatorname{div} u_{t}\right) \psi+\nabla p \nabla \psi\right) \mathrm{d} x & =\int_{\Omega} h \psi \mathrm{d} x+\int_{\partial \Omega} \gamma(x)\left(p^{*}-p\right) \psi \mathrm{d} s(x)
\end{aligned}
$$

for every test functions $\phi \in W_{0}^{1,2}\left(\Omega ; \mathbb{R}^{3}\right)$ and $\psi \in W^{1,2}(\Omega)$.

With the intention to use the Galerkin method, we choose $\left\{\phi_{k} ; k=1,2, \ldots\right\}$ in $L^{2}\left(\Omega ; \mathbb{R}^{3}\right)$ and $\left\{\psi_{l} ; l=0,1,2, \ldots\right\}$ in $L^{2}(\Omega)$ to be the complete orthonormal systems of eigenfunctions defined by

$-\operatorname{div} \mathbf{A} \nabla_{s} \phi_{k}=\lambda_{k} \phi_{k}$ in $\Omega,\left.\quad \phi_{k}\right|_{\partial \Omega}=0, \quad-\Delta \psi_{l}=\mu_{l} \psi_{l}$ in $\Omega,\left.\quad \nabla \psi_{l} \cdot n\right|_{\partial \Omega}=0$,

with $\mu_{0}=0, \lambda_{k}>0, \mu_{l}>0$ for $k, l \geq 1$.

\subsection{Galerkin approximations}

Approximate $2 \pi$-periodic solutions will be searched in the form

$$
u^{(m)}(x, t)=\sum_{j=-m}^{m} \sum_{k=1}^{m} u_{j k} e_{j}(t) \phi_{k}(x), \quad p^{(m)}(x, t)=\sum_{j=-m}^{m} \sum_{l=0}^{m} p_{j l} e_{j}(t) \psi_{l}(x),
$$

with

$$
e_{j}(t)= \begin{cases}\sin j t & \text { if } j \geq 1 \\ \cos j t & \text { if } j \leq 0\end{cases}
$$

and with real coefficients $u_{j k}, p_{j l}$ which satisfy the system

$$
\begin{aligned}
\int_{2 \pi}^{4 \pi} & \int_{\Omega}\left(\left(u_{t t}^{(m)}+u_{t}^{(m)}\right) \phi_{k}(x)+\mathbf{A} \nabla_{s} u^{(m)}: \nabla_{s} \phi_{k}(x)+p^{(m)} \operatorname{div} \phi_{k}(x)\right) e_{j}(t) \mathrm{d} x \mathrm{~d} t \\
& =\int_{2 \pi}^{4 \pi} \int_{\Omega} f \phi_{k}(x) e_{j}(t) \mathrm{d} x \mathrm{~d} t \\
\int_{2 \pi}^{4 \pi} & \int_{\Omega}\left(\left(G_{R}\left[p^{(m)}\right]_{t}-\operatorname{div} u_{t}^{(m)}\right) \psi_{l}(x)+\nabla p^{(m)} \nabla \psi_{l}(x)\right) e_{j}(t) \mathrm{d} x \mathrm{~d} t \\
= & \int_{2 \pi}^{4 \pi} \int_{\Omega} h \psi_{l}(x) e_{j}(t) \mathrm{d} x \mathrm{~d} t+\int_{2 \pi}^{4 \pi} \int_{\partial \Omega} \gamma(x)\left(p^{*}-p^{(m)}\right) \psi_{l}(x) e_{j}(t) \mathrm{d} s(x) \mathrm{d} t .
\end{aligned}
$$


for $j=-m, \ldots, m, k=1, \ldots, m, l=0, \ldots, m$. This is an algebraic problem of $(2 m+1)^{2}$ equations for a $(2 m+1)^{2}$-dimensional real unknown vector

$$
U=\left(u_{j k}, p_{j l}\right), j=-m, \ldots, m, k=1, \ldots, m, l=0, \ldots, m
$$

that we solve by using the degree theory. We define a continuous family of mappings $\mathcal{T}_{\alpha}$ : $\mathbb{R}^{(2 m+1)^{2}} \rightarrow \mathbb{R}^{(2 m+1)^{2}}$ for $\alpha \in[0,1]$ which with $U$ as in (4.7) associate the vector $\mathcal{T}_{\alpha}(U)=V$ of the form

$$
V=\left(v_{j k}, w_{j l}\right), j=-m, \ldots, m, k=1, \ldots, m, l=0, \ldots, m,
$$

given by the formula

$$
\begin{aligned}
v_{j k}= & \int_{2 \pi}^{4 \pi} \int_{\Omega}\left(\left(u_{t t}^{(m)}+u_{t}^{(m)}\right) \phi_{k}(x)+\mathbf{A} \nabla_{s} u^{(m)}: \nabla_{s} \phi_{k}(x)+p^{(m)} \operatorname{div} \phi_{k}(x)\right) e_{j}(t) \mathrm{d} x \mathrm{~d} t \\
& -\int_{2 \pi}^{4 \pi} \int_{\Omega} \alpha f \phi_{k}(x) e_{j}(t) \mathrm{d} x \mathrm{~d} t \\
w_{j l}= & \left.\int_{2 \pi}^{4 \pi} \int_{\Omega}\left(\left((1-\alpha) p^{(m)}+\alpha G_{R}\left[p^{(m)}\right]\right)_{t}-\operatorname{div} u_{t}^{(m)}\right) \psi_{l}(x)+\nabla p^{(m)} \nabla \psi_{l}(x)\right) e_{j}(t) \mathrm{d} x \mathrm{~d} t \\
& -\int_{2 \pi}^{4 \pi} \int_{\Omega} \alpha h \psi_{l}(x) e_{j}(t) \mathrm{d} x \mathrm{~d} t-\int_{2 \pi}^{4 \pi} \int_{\partial \Omega} \gamma(x)\left(\alpha p^{*}-p^{(m)}\right) \psi_{l}(x) e_{j}(t) \mathrm{d} s(x) \mathrm{d} t .
\end{aligned}
$$

System (4.5)-(4.6) can be interpreted as $\mathcal{T}_{1}(U)=0$. Clearly, all mappings $\mathcal{T}_{\alpha}$ for $\alpha \in[0,1]$ are continuous. We now show that the equation $\mathcal{T}_{\alpha}(U)=0$ for any $\alpha \in[0,1]$ has no solution $U$ on the boundary of a sufficiently large ball $\mathcal{B}_{K} \subset \mathbb{R}^{(2 m+1)^{2}}$. Indeed, assume that $V=\mathcal{T}_{\alpha}(U)=0$ for some $U$ and $\alpha$. Note that

$$
u_{t}^{(m)}(x, t)=\sum_{k=1}^{m} \sum_{j=-m}^{m} \hat{u}_{j k} e_{j}(t) \phi_{k}(x) \text { with } \hat{u}_{j k}=j u_{-j k} .
$$

We then have

$$
\begin{aligned}
0= & \sum_{k=1}^{m} \sum_{j=-m}^{m} v_{j k} \hat{u}_{j k}+\sum_{l=0}^{m} \sum_{j=-m}^{m} w_{j l} p_{j l} \\
= & \int_{2 \pi}^{4 \pi} \int_{\Omega}\left(\left|u_{t}^{(m)}\right|^{2}-\alpha f u_{t}^{(m)}+\alpha G_{R}\left[p^{(m)}\right]_{t} p^{(m)}+\left|\nabla p^{(m)}\right|^{2}-\alpha h p^{(m)}\right) \mathrm{d} x \mathrm{~d} t \\
& -\int_{2 \pi}^{4 \pi} \int_{\partial \Omega} \gamma(x)\left(\alpha p^{*}-p^{(m)}\right) p^{(m)} \mathrm{d} s(x) \mathrm{d} t,
\end{aligned}
$$

and using (3.16) we obtain

$$
\begin{aligned}
& \int_{2 \pi}^{4 \pi} \int_{\Omega}\left(\left|u_{t}^{(m)}\right|^{2}+\left|\nabla p^{(m)}\right|^{2}\right) \mathrm{d} x \mathrm{~d} t+\int_{2 \pi}^{4 \pi} \int_{\partial \Omega} \gamma(x)\left|p^{(m)}\right|^{2} \mathrm{~d} s(x) \mathrm{d} t \\
& \quad \leq \alpha \int_{2 \pi}^{4 \pi} \int_{\Omega}\left(f u_{t}^{(m)}+h p^{(m)}\right) \mathrm{d} x \mathrm{~d} t+\alpha \int_{2 \pi}^{4 \pi} \int_{\partial \Omega} \gamma(x) p^{*} p^{(m)} \mathrm{d} s(x) \mathrm{d} t
\end{aligned}
$$

so that

$$
\sum_{k=1}^{m} \sum_{j=-m}^{m}\left|u_{j k}\right|^{2}+\sum_{l=0}^{m} \sum_{j=-m}^{m}\left|p_{j l}\right|^{2} \leq K
$$


independently of $\alpha$. We see that $\mathcal{T}_{\alpha}$ is a homotopy of continuous mappings on $\mathbb{R}^{(2 m+1)^{2}}$ and such that the equation $\mathcal{T}_{\alpha}(U)=0$ has no solution on the boundary of any ball of radius bigger than $K$. Since $\mathcal{T}_{0}$ is odd, its topological degree with respect to the ball $\mathcal{B}_{K+1}$ and the point 0 is nonzero, and remains constant for all $\alpha \in[0,1]$. We conclude that the equation $\mathcal{T}_{1}(U)=0$ has a solution, which, by definition satisfies (4.5)-(4.6), as well as the estimate

$$
\left\|u_{t}^{(m)}\right\|_{2, \Omega, 2 \pi}+\left\|\nabla p^{(m)}\right\|_{2, \Omega, 2 \pi}+\left\|p^{(m)}\right\|_{2, \partial \Omega, 2 \pi, \gamma} \leq C \delta
$$

with $\delta$ from Theorem 2.1 and with a constant $C$ independent of $m$ and $\delta$.

By iterating the formula (4.11) we obtain

$$
\begin{aligned}
& u_{t t t}^{(m)}(x, t)=\sum_{k=1}^{m} \sum_{j=-m}^{m} u_{j k}^{\sharp} e_{j}(t) \phi_{k}(x) \text { with } u_{j k}^{\sharp}=-j^{3} u_{-j k}, \\
& p_{t t}^{(m)}(x, t)=\sum_{l=0}^{m} \sum_{j=-m}^{m} p_{j l}^{\sharp} e_{j}(t) \psi_{l}(x) \text { with } p_{j l}^{\sharp}=-j^{2} p_{j l} .
\end{aligned}
$$

We now test (4.5) by $-u_{j k}^{\sharp}$, (4.6) by $-p_{j l}^{\sharp}$, and use (3.15) to obtain

$$
\left\|u_{t t}^{(m)}\right\|_{2, \Omega, 2 \pi}^{2}+\left\|p_{t}^{(m)}\right\|_{3, \Omega, 2 \pi}^{3}+\left\|\nabla p_{t}^{(m)}\right\|_{2, \Omega, 2 \pi}^{2}+\left\|p_{t}^{(m)}\right\|_{2, \partial \Omega, 2 \pi, \gamma}^{2} \leq C \delta^{2}
$$

with a constant $C$ independent of $m$ and $\delta$. Then, testing (4.5) by $\bar{u}_{j k}:=-j^{2} u_{j k}$, we obtain using (4.12), (4.13) that

$$
\left\|\nabla_{s} u_{t}^{(m)}\right\|_{2, \Omega, 2 \pi} \leq C \delta
$$

By (3.17) we have

$$
\left|G_{R}\left[p^{(m)}\right]_{t}(x, t)\right| \leq H_{\rho}\left\|p^{(m)}(x, \cdot)\right\|_{[2 \pi, 4 \pi]}\left|p_{t}^{(m)}(x, t)\right|
$$

for all $x \in \Omega$ and $t \in[2 \pi, 4 \pi]$. Using the inequality

$$
\left\|p^{(m)}(x, \cdot)\right\|_{[2 \pi, 4 \pi]} \leq \frac{1}{2 \pi} \int_{2 \pi}^{4 \pi}\left|p^{(m)}(x, \tau)\right| \mathrm{d} \tau+\int_{2 \pi}^{4 \pi}\left|p_{t}^{(m)}(x, \tau)\right| \mathrm{d} \tau,
$$

we have, by (4.16) and Minkowski's inequality,

$$
\left(\int_{\Omega}\left\|p^{(m)}(x, \cdot)\right\|_{[2 \pi, 4 \pi]}^{6} \mathrm{~d} x\right)^{1 / 6} \leq C \int_{2 \pi}^{4 \pi}\left(\int_{\Omega}\left(\left|p^{(m)}(x, t)\right|^{6}+\left|p_{t}^{(m)}(x, t)\right|^{6}\right) \mathrm{d} x\right)^{1 / 6} \mathrm{~d} t,
$$

so that, by the Sobolev embedding and estimates (4.12)-(4.13),

$$
\begin{aligned}
& \left(\int_{\Omega}\left\|p^{(m)}(x, \cdot)\right\|_{[2 \pi, 4 \pi]}^{6} \mathrm{~d} x\right)^{1 / 6} \\
& \leq C \int_{2 \pi}^{4 \pi}\left(\int_{\Omega}\left(\left|p^{(m)}(x, t)\right|^{2}+\left|p_{t}^{(m)}(x, t)\right|^{2}+\left|\nabla p^{(m)}(x, t)\right|^{2}+\left|\nabla p_{t}^{(m)}(x, t)\right|^{2}\right) \mathrm{d} x\right)^{1 / 2} \mathrm{~d} t \leq C \delta .
\end{aligned}
$$

From (4.15) and Hölder's inequality it follows

$$
\int_{\Omega}\left|G_{R}\left[p^{(m)}\right]_{t}(x, t)\right|^{2} \mathrm{~d} x \leq C \delta^{2}\left(\int_{\Omega}\left|p_{t}^{(m)}(x, t)\right|^{3} \mathrm{~d} x\right)^{2 / 3}
$$

hence

$$
\int_{2 \pi}^{4 \pi}\left(\int_{\Omega}\left|G_{R}\left[p^{(m)}\right]_{t}(x, t)\right|^{2} \mathrm{~d} x\right)^{3 / 2} \mathrm{~d} t \leq C \delta^{5} .
$$




\subsection{Passage to the limit}

The compactness argument will be based on an anisotropic embedding formula which is a special case of the theory developed in [10]. For a bounded domain $D \subset \mathbb{R}^{N}$, an open bounded interval $\omega \subset \mathbb{R}$, and real numbers $q, r \geq 1$ we define for $v \in L^{r}\left(\omega ; L^{q}(D)\right)$ and $w \in L^{q}\left(D ; L^{r}(\omega)\right)$ their anisotropic norms

$$
\|v\|_{q, r}=\left(\int_{\omega}\left(\int_{D}|v(x, t)|^{q} \mathrm{~d} x\right)^{r / q} \mathrm{~d} t\right)^{1 / r}, \quad\|w\|_{r, q}^{*}=\left(\int_{D}\left(\int_{\omega}|w(x, t)|^{r} \mathrm{~d} t\right)^{q / r} \mathrm{~d} x\right)^{1 / q} .
$$

We also introduce the anisotropic Sobolev spaces

$$
\begin{aligned}
& W^{r_{0}, q_{0} ; r_{1}, q_{1}}(\omega, D)=\left\{v \in L^{1}(D \times \omega): \frac{\partial v}{\partial t} \in L^{r_{0}}\left(\omega ; L^{q_{0}}(D)\right), \nabla v \in L^{r_{1}}\left(\omega ; L^{q_{1}}(D)\right)\right\} \\
& W^{q_{0}, r_{0} ; q_{1}, r_{1}}(D, \omega)=\left\{w \in L^{1}(D \times \omega): \frac{\partial w}{\partial t} \in L^{q_{0}}\left(D ; L^{r_{0}}(\omega)\right), \nabla w \in L^{q_{1}}\left(D ; L^{r_{1}}(\omega)\right)\right\} .
\end{aligned}
$$

We will repeatedly use the following compact embedding result.

Proposition 4.1. Let the domain D be Lipschitzian.

(i) If $q \geq \max \left\{q_{0}, q_{1}\right\}, r \geq \max \left\{r_{0}, r_{1}\right\}$, and

$$
\left(1-\frac{1}{r_{0}}+\frac{1}{r}\right)\left(\frac{1}{N}-\frac{1}{q_{1}}+\frac{1}{q}\right)>\left(\frac{1}{r_{1}}-\frac{1}{r}\right)\left(\frac{1}{q_{0}}-\frac{1}{q}\right),
$$

then $W^{r_{0}, q_{0} ; r_{1}, q_{1}}(\omega, D)$ is compactly embedded in $L^{r}\left(\omega ; L^{q}(D)\right)$ and $W^{q_{0}, r_{0} ; q_{1}, r_{1}}(D, \omega)$ is compactly embedded in $L^{q}\left(D ; L^{r}(\omega)\right)$.

(ii) If $q \geq \max \left\{q_{0}, q_{1}\right\}$ is such that

$$
\left(1-\frac{1}{r_{0}}\right)\left(\frac{1}{N}-\frac{1}{q_{1}}+\frac{1}{q}\right)>\frac{1}{r_{1}}\left(\frac{1}{q_{0}}-\frac{1}{q}\right)
$$

then $W^{q_{0}, r_{0} ; q_{1}, r_{1}}(D, \omega)$ is compactly embedded in $L^{q}(D ; C(\bar{\omega}))$.

(iii) If

$$
\left(1-\frac{1}{r_{0}}\right)\left(\frac{1}{N}-\frac{1}{q_{1}}\right)>\frac{1}{r_{1} q_{0}}
$$

then $W^{q_{0}, r_{0} ; q_{1}, r_{1}}(D, \omega)$ is compactly embedded in $\left.C(\bar{D} \times \bar{\omega})\right)$.

We have bounds independent of $m$ in $L^{3}\left(\Omega ; L^{3}(2 \pi, 4 \pi)\right)$ for $p_{t}^{(m)}$ and in $L^{2}\left(\Omega ; L^{r}(2 \pi, 4 \pi)\right)$ for $\nabla p^{(m)}$ for all $r>1$ by virtue of (4.12) and (4.13). Hence, by Proposition 4.1 (ii), $\left\{p^{(m)}\right\}$ is a compact sequence in $L^{q}(\Omega ; C[2 \pi, 4 \pi])$ for each $q \in[1,6)$. Furthermore, by (4.18) $\left\{G_{R}\left[p^{(m)}\right]_{t}\right\}$ 
is a bounded sequence in $L^{3}\left((2 \pi, 4 \pi) ; L^{2}(\Omega)\right)$. By (3.2) and (3.17), we can select a subsequence (still indexed by $m$ ) in such a way that

$$
\left\{\begin{array}{lll}
p^{(m)} \rightarrow p & \text { strongly in } & L^{4}(\Omega ; C[2 \pi, 4 \pi]) \\
G_{R}\left[p^{(m)}\right] \rightarrow G_{R}[p] & \text { strongly in } & L^{2}(\Omega ; C[2 \pi, 4 \pi]) \\
G_{R}\left[p^{(m)}\right]_{t} \rightarrow G_{R}[p]_{t} & \text { weakly in } & L^{3}\left((2 \pi, 4 \pi) ; L^{2}(\Omega)\right) .
\end{array}\right.
$$

All the other terms in (4.5)-(4.6) are linear, so that we can pass to the weak limit and conclude that the system

$$
\begin{aligned}
\int_{2 \pi}^{4 \pi} & \int_{\Omega}\left(\left(u_{t t}+u_{t}\right) \Phi(x, t)+\mathbf{A} \nabla_{s} u: \nabla_{s} \Phi(x, t)+p \operatorname{div} \Phi(x, t)\right) \mathrm{d} x \mathrm{~d} t \\
& =\int_{2 \pi}^{4 \pi} \int_{\Omega} f \Phi(x, t) \mathrm{d} x \mathrm{~d} t \\
\int_{2 \pi}^{4 \pi} & \int_{\Omega}\left(\left(G_{R}[p]_{t}-\operatorname{div} u_{t}\right) \Psi(x, t)+\nabla p \nabla \Psi(x, t)\right) \mathrm{d} x \mathrm{~d} t \\
= & \int_{2 \pi}^{4 \pi} \int_{\Omega} h \Psi(x, t) \mathrm{d} x \mathrm{~d} t+\int_{2 \pi}^{4 \pi} \int_{\partial \Omega} \gamma(x)\left(p^{*}-p\right) \Psi(x, t) \mathrm{d} s(x) \mathrm{d} t,
\end{aligned}
$$

is satisfied for all arbitrarily chosen $2 \pi$-periodic test functions $\Phi \in L^{2}\left((2 \pi, 4 \pi) ; W_{0}^{1,2}\left(\Omega ; \mathbb{R}^{3}\right)\right)$, $\Psi \in L^{2}\left((2 \pi, 4 \pi) ; W^{1,2}(\Omega)\right)$, with the regularity $u, u_{t}, u_{t t}, \nabla_{s} u_{t}, \nabla p, \nabla p_{t} \in L_{2 \pi}^{2}(\Omega), p_{t} \in L_{2 \pi}^{3}(\Omega)$, $G_{R}[p]_{t} \in L^{3}\left((2 \pi, 4 \pi) ; L^{2}(\Omega)\right)$. Choosing $\Psi$ with compact support in $\Omega$, we obtain from (4.14), (4.18) that

$$
\|\Delta p\|_{2, \Omega, 2 \pi} \leq C \delta
$$

and similarly

$$
\left\|\operatorname{div} \mathbf{A} \nabla_{s} u\right\|_{2, \Omega, 2 \pi} \leq C \delta
$$

so that the identities

$$
\begin{aligned}
u_{t t}+u_{t}-\operatorname{div} \mathbf{A} \nabla_{s} u & =\nabla p+f, \\
G_{R}[p]_{t}-\Delta p & =\operatorname{div} u_{t}+h
\end{aligned}
$$

with boundary conditions (2.3) hold in the sense of $L_{2 \pi}^{2}(\Omega)$.

For $s>0$, and a function $v \in L_{2 \pi}^{2}(\Omega)$ such that $v_{t} \in L_{2 \pi}^{2}(\Omega)$, we define

$$
v^{(\tau)}(x, t)=\frac{1}{\tau}(v(x, t+\tau)-v(x, t)) .
$$

We have indeed

$$
\int_{2 \pi}^{4 \pi} \int_{\Omega}\left|v^{(\tau)}(x, t)\right|^{2} \mathrm{~d} x \mathrm{~d} t \leq \int_{2 \pi}^{4 \pi} \int_{\Omega}\left|v_{t}(x, t)\right|^{2} \mathrm{~d} x \mathrm{~d} t, \quad \lim _{s \rightarrow 0} \int_{2 \pi}^{4 \pi} \int_{\Omega}\left|v^{(\tau)}(x, t)-v_{t}(x, t)\right|^{2} \mathrm{~d} x \mathrm{~d} t=0 .
$$

Put $\hat{f}=\nabla p+f$. Then $\hat{f}, \hat{f}_{t} \in L_{2 \pi}^{2}(\Omega)$ by virtue of (4.13) and the hypotheses on $f$. From (4.23) it follows for $s>0$ that

$$
u_{t t}^{(\tau)}+u_{t}^{(\tau)}-\operatorname{div} \mathbf{A} \nabla_{s} u^{(\tau)}=\hat{f}^{(\tau)} .
$$


We test (4.26) by $u_{t}^{(\tau)}$ and obtain for a. e. $t \in \mathbb{R}$ that

$$
\frac{\mathrm{d}}{\mathrm{d} t} \int_{\Omega}\left(\left|u_{t}^{(\tau)}\right|^{2}+\mathbf{A} \nabla_{s} u^{(\tau)}: \nabla_{s} u^{(\tau)}\right)(x, t) \mathrm{d} x+\int_{\Omega}\left|u_{t}^{(\tau)}\right|^{2}(x, t) \mathrm{d} x \leq \int_{\Omega}\left|\hat{f}^{(\tau)}\right|^{2}(x, t) \mathrm{d} x,
$$

so that

$$
\begin{aligned}
& \frac{\mathrm{d}}{\mathrm{d} t} \int_{\Omega}\left(\left|u_{t}^{(\tau)}\right|^{2}+\mathbf{A} \nabla_{s} u^{(\tau)}: \nabla_{s} u^{(\tau)}\right)(x, t) \mathrm{d} x+\int_{\Omega}\left(\left|u_{t}^{(\tau)}\right|^{2}+\mathbf{A} \nabla_{s} u^{(\tau)}: \nabla_{s} u^{(\tau)}\right)(x, t) \mathrm{d} x \\
& \quad \leq \int_{\Omega}\left(\left|\hat{f}^{(\tau)}\right|^{2}+\mathbf{A} \nabla_{s} u^{(\tau)}: \nabla_{s} u^{(\tau)}\right)(x, t) \mathrm{d} x .
\end{aligned}
$$

Put

$y(t)=\int_{\Omega}\left(\left|u_{t}^{(\tau)}\right|^{2}+\mathbf{A} \nabla_{s} u^{(\tau)}: \nabla_{s} u^{(\tau)}\right)(x, t) \mathrm{d} x, \quad \beta(t)=\int_{\Omega}\left(\left|\hat{f}^{(\tau)}\right|^{2}+\mathbf{A} \nabla_{s} u^{(\tau)}: \nabla_{s} u^{(\tau)}\right)(x, t) \mathrm{d} x$.

Both $y$ and $\beta$ belong to $L_{2 \pi}^{1}$ by (4.13) - (4.14) and

$$
\int_{2 \pi}^{4 \pi}(y(t)+\beta(t)) \mathrm{d} t \leq C \delta^{2} .
$$

Then (4.27) is an inequality of the form

$$
\frac{\mathrm{d}}{\mathrm{d} t} y(t)+y(t) \leq \beta(t)
$$

which implies by the Gronwall argument that

$$
y(t) \leq C \int_{2 \pi}^{4 \pi}(y(\tau)+\beta(\tau)) \mathrm{d} \tau \leq C \delta^{2} \quad \text { for a. e. } t \in \mathbb{R},
$$

and we conclude by passing to the limit as $s \rightarrow 0$ and using (4.25) that $u_{t t}, \nabla_{s} u_{t}$ belong to $L^{\infty}\left(\mathbb{R} ; L^{2}(\Omega)\right)$ with

$$
\sup _{t \in \mathbb{R}} \operatorname{ess} \int_{\Omega}\left(\left|u_{t t}\right|^{2}+\left|\nabla_{s} u_{t}\right|^{2}\right)(x, t) \mathrm{d} x \leq C \delta^{2} .
$$

We see that in (4.24), we have $h \in L^{\infty}\left(\mathbb{R} ; L^{2}(\Omega)\right)$ by hypothesis, $\operatorname{div} u_{t} \in L^{\infty}\left(\mathbb{R} ; L^{2}(\Omega)\right)$ by Korn's inequality and (4.29), and $G_{R}[p]_{t} \in L^{3}\left((2 \pi, 4 \pi) ; L^{2}(\Omega)\right)$ by (4.20), with bounds proportional to $\delta$. Hence, with the notation (4.19),

$$
\|\Delta p\|_{2,3} \leq C \delta
$$

By hypotheses about the regularity of $\partial \Omega$ and of the boundary data, and by (4.13), we have

$$
\left\|\frac{\partial^{2} p}{\partial x_{i} \partial x_{j}}\right\|_{2,3} \leq C \delta, \quad\left\|\frac{\partial^{2} p}{\partial x_{i} \partial t}\right\|_{2,2} \leq C \delta
$$

for all $i, j=1,2,3$. From Proposition 4.1(i) we obtain that

$$
\|\nabla p\|_{r, r} \leq C \delta
$$


for $r<14 / 3$. Combining this result with the fact that

$$
\left\|p_{t}\right\|_{3,3} \leq C \delta
$$

which follows from (4.13), we can use Proposition 4.1(iii) and conclude that

$$
\max \{|p(x, t)|:(x, t) \in \bar{\Omega} \times[2 \pi .4 \pi]\} \leq C \delta
$$

provided $r>9 / 2$. Thus, choosing

$$
r \in\left(\frac{9}{2}, \frac{14}{3}\right)
$$

we see that if $\delta>0$ is chosen sufficiently small, then $|p(x, t)|$ does not exceed the critical value $R$, and from (3.18) we infer that the solution of (4.23)-(4.24) that we have constructed is the desired solution of (2.1)-(2.2), which we wanted to prove.

\section{Conclusion}

We have proved that a model for the propagation of periodic mechanical waves inside an elastic partially saturated porous body with capillary hysteresis represented by a Preisach operator is well posed under periodic boundary forcing provided the boundary forces are sufficiently small. The meaning of the smallness condition is to keep the pressure values within the convexity domain of the Preisach operator and exploit the hysteresis second order energy inequality.

\section{References}

[1] Albers B. Monochromatic surface waves at the interface between poroelastic and fluid halfspaces. Proc. Royal Soc. A. 2006; 462: 701-723.

[2] Albers B. Modeling the hysteretic behavior of the capillary pressure in partially saturated porous media - a review. Acta Mechanica. 2014; 225 (8): 2163-2189.

[3] Albers B. Main drying and wetting curves of soils - on measurements, prediction and influence on wave propagation. Engineering Transactions. 2015; 63 (1): 5-34.

[4] Albers B. On the influence of the hysteretic behavior of the capillary pressure on the wave propagation in partially saturated soils. To appear in Journal of Physics: Conference Series, Proceedings of the 7th International Workshop on Multi-Rate Processes and Hysteresis. 2014.

[5] Albers B. Modeling and Numerical Analysis of Wave Propagation in Saturated and Partially Saturated Porous Media. Habilitation Thesis. Veröffentlichungen des Grundbauinstitutes der Technischen Universität Berlin, Shaker: Aachen; 2010.

[6] Albers B., Krejčí P. Unsaturated porous media flow with thermomechanical interaction. Accepted for publication in MMAS.

[7] Albers B., Krejčí P. Hysteresis in unsaturated porous media - two models for wave propagation and engineering applications. In Continuous Media with Microstructure 2, Eds. B. Albers and M. Kuczma, Springer, 2016: 217-229. 
[8] Bagagiolo F, Visintin A. Hysteresis in filtration through porous media. Z. Anal. Anwendungen. 2000; 19 (4): 977-997.

[9] Bagagiolo F, Visintin A. Porous media filtration with hysteresis. Adv. Math. Sci. Appl. 2004; 14 (2): 379-403.

[10] Besov OV, Il'in VP, Nikol'skiu SM. Integral Representations of Functions and Imbedding Theorems. Scripta Series in Mathematics. Halsted Press (John Wiley \& Sons): New York-Toronto, Ont.London; 1978 (Vol. I), 1979 (Vol. II). Russian version Nauka: Moscow; 1975.

[11] Cosentini RM, Foti S. Evaluation of porosity and degree of saturation from seismic and electrical data. Géotechnique 2014; 64 (4): 278-286.

[12] Eleuteri M, Kopfová J, Krejčí P. Magnetohydrodynamic flow with hysteresis. SIAM J. Math. Anal. 2009; 41: 435-464.

[13] Flynn D. Modelling the flow of water through multiphase porous media with the Preisach model. PhD Thesis. University College Cork; 2008.

[14] Flynn D, McNamara H, O'Kane JP, Pokrovskiu AV. Application of the Preisach model to soilmoisture hysteresis. The Science of Hysteresis, Volume 3, Eds. Bertotti G, Mayergoyz I. Academic Press: Oxford; 2006: 689-744.

[15] Foti S, Lai CG, Rix, GJ, Strobbia C. Surface Wave Methods for Near-Surface Site Characterization. Boca Raton: CRC Press; 2014.

[16] Gan WS. Acoustical Imaging: Techniques and Applications for Engineers. Wiley; 2012.

[17] Haverkamp R, Reggiani P, Ross PJ, Parlange J-Y. Soil water hysteresis prediction model based on theory and geometric scaling. Environmental Mechanics, Water, Mass and Engergy Transfer in the Biosphere. Eds. Raats PAC, Smiles D, Warrick AW. American Geophysical Union: 2002; 213-246.

[18] Krasnosel'skiu MA, Pokrovskiı̌ AV. Systems with Hysteresis. Springer: Berlin; 1989. Russian edition: Nauka: Moscow; 1983.

[19] Krautkrämer J, Krautkrämer H. Ultrasonic Testing of Materials. Springer-Verlag, Berlin; 1990.

[20] Krejčí P. Hysteresis, Convexity and Dissipation in Hyperbolic Equations. Gakuto Intern. Ser. Math. Sci. Appl. Vol. 8. Gakkotōsho: Tokyo; 1996.

[21] Krejčí P. On Maxwell equations with the Preisach hysteresis operator: the one-dimensional timeperiodic case. Apl. Mat. 1989; 34: 364-374.

[22] Preisach F. Über die magnetische Nachwirkung. Z. Phys. 1935; 94: 277-302 (in German).

[23] Roesset JM. Nondestructive dynamic testing of soils and pavements. Tamkang Journal of Science and Engineering 1998; 61-81.

[24] Shull PJ. Nondestructive Evaluation: Theory, Techniques, and Applications. Marcel Dekker Inc.; 2002.

[25] Veenstra M. White DJ, Schaefer VR. Synthesis of Nondestructive Testing Technologies for Geomaterial Applications. Iowa State University; 2005. 